\title{
Fitonematoides associados à cultura da banana em sistemas cacau cabruca
}

Carla da Silva Sousa ${ }^{*}$, Joseane Santos da Silva² Érica Conceição de Lima $^{3}$

\author{
${ }^{1}$ Doutora Tecnologias Energéticas e \\ Nucleares. Docente do Instituto \\ Federal de Educação, Ciência e \\ Tecnologia Baiano, Campus Uruçuca. \\ https://orcid.org/0000-0003-4381- \\ $\underline{0524}$ \\ ${ }^{2}$ Discente do Curso Superior de \\ Tecnologia em Agroecologia do \\ Instituto Federal de Educação, \\ Ciência e Tecnologia Baiano, campus \\ Uruçuca. \\ https://orcid.org/0000-0002-6425- \\ $\underline{585 X}$ \\ ${ }^{3}$ Discente do Curso Superior de \\ Tecnologia em Agroecologia do \\ Instituto Federal de Educação, \\ Ciência e Tecnologia Baiano, campus \\ Uruçuca. \\ https://orcid.org/0000-0003-1971- \\ $\underline{3484}$
}

*Autor correspondente: carla.sousa@ifbaiano.edu.br

\begin{abstract}
Resumo:
A banana é uma das frutas mais consumidas no mundo, e ganhou destaque na região do Sul da Bahia, quando foi escolhida para ser a cultura inserida para o sombreamento do cacau em sistemas agroflorestais. Entretanto, problemas fitossanitários, destacandose os fitonematoides reduzem a produtividade e influenciam na qualidade dos frutos. Neste sentido, o presente estudo, teve como objetivo realizar o levantamento de fitonematoides associados a cultura da bananeira em sistemas agroflorestais de cacau cabruca nos municípios de Uruçuca e Itajuípe localizados na Região Sul da Bahia. Foram realizadas coletas de amostras de solo e raízes das plantas de bananeira em 10 áreas de cacau cabruca, para quantificação e identificação das espécies de fitonematoides. $\mathrm{O}$ nematoide Helicotylenchus multicinctus e os nematoides de vida livre foram registrados nas amostras de solo e raízes em todas as áreas (frequência relativa de 100\%). O nematoide Rotylenchus reniformis foi registrado nas amostras de solo coletadas nas áreas Uruçuca 8, Itajuípe 1 e Itajuípe 2, com populações de 10, 30 e 40 nematoides $/ 250 \mathrm{~cm}^{3}$ de solo, respectivamente. Este nematoide foi observado apenas em amostras de raízes coletadas na área Itajuípe 2 (20 nematoides/10 g de raízes). Embora seja considerado, a principal espécie de fitonematoides causador de danos econômicos à cultura da banana, o Radopholus similis foi observado apenas em amostras de solo coletadas na área Uruçuca 7, com população de 10 nematoides/10g de raízes.
\end{abstract}

Palavras chave: Musa spp., Fitonematoses, Sistemas agroflorestais.

\section{REVISTA MACAMBIRA}

Instituto Federal de Educação, Ciência e Tecnologia Baiano, Campus Serrinha. Estrada Vicinal de Aparecida, s/n, Bairro Aparecida, Serrinha (Ba), CEP: 48700-000, sala 01, prédio acadêmico. 


\title{
Phytonematoids associated with banana culture in cocoa cabruca systems
}

\author{
Carla da Silva Sousa ${ }^{1}$, Joseane Santos da Silva² ${ }^{2}$ Érica Conceição de Lima ${ }^{3}$
}

\author{
${ }^{1}$ Professor at the Federal Institute \\ of Education, Science and \\ Technology Baiano, Campus \\ Uruçuca. PhD in Energy and \\ Nuclear Technologies. \\ https://orcid.org/0000-0003-4381- \\ $\underline{0524}$ \\ ${ }^{2}$ Student Course in Technology in \\ Agroecology, Federal Institute of \\ Education, Science and \\ Technology Baiano, Campus \\ Uruçuca. \\ https://orcid.org/0000-0002-6425- \\ $\underline{585 \mathrm{X}}$ \\ ${ }^{3}$ Student Course in Technology in \\ Agroecology, Federal Institute of \\ Education, Science and \\ Technology Baiano, Campus \\ Uruçuca. \\ https://orcid.org/0000-0003-1971- \\ $\underline{3484}$
}

*Corresponding author: carla.sousa@ifbaiano.edu.br

\begin{abstract}
:
Banana is one of the most consumed fruits in the world, gaining prominence in the southern region of Bahia, when it was chosen as the crop inserted for the shading of cocoa in agroforestry systems. However, phytosanitary problems, especially phytomatoids, reduce productivity and influence fruit quality. In doing so, this study aimed to conducted an analysis of the occurrence and diversity of phytomatoids associated with banana cultivation in agroforestry systems of cocoa cabruca in Uruçuca and Itajuípe in the Southern Region of Bahia. Soil and root samples of banana plants were collected in cabruca cocoa plantations in order to quantify and identify the phytomatoid species. Helicotylenchus multicinctus nematodes and free-living nematodes were found in the soil and root samples in all plantations (relative frequency of 100\%). The nematode Rotylenchus reniformis was observed in soil samples collected in Uruçuca 8, Itajuípe 1 and Itajuípe 2 areas, with populations of 10,30 and 40 nematodes $/ 250 \mathrm{~cm}^{3}$ of soil, respectively. This nematode was observed only in root samples collected in the Itajuípe 2 area (20 nematodes/10 g of roots). Although it is considered the main species of phytomatoids causing economic consequences to banana crop, Radopholus similis was observed only in soil samples collected in Uruçuca 7 area, with a population of 10 nematodes $/ 10 \mathrm{~g}$ of roots.
\end{abstract}

Keywords: Musa spp., Fitonematoses, Agroforestry systems.

MACAMBIRA JOURNAL

Federal Institute of Education, Science and Technology Baiano, Campus Serrinha. Estrada Vicinal de Aparecida, s/n, Bairro Aparecida, Serrinha, Bahia, Brasil, CEP: 48700-000, sala 01, prédio acadêmico. 


\section{Introdução}

A Mata Atlântica tem como principal característica a grande variedade de espécies faunísticas e florísticas, bem como, uma expressiva complexidade ecológica e grau de ameaça e endemismo, tornandoa um Hotspot para a conservação da biodiversidade (OLIVEIRA et al., 2011). Entretanto, existe apenas $1 \%$ a $5 \%$ da cobertura original, resultado da redução de espécies arbóreas, bem como, do elevado grau de degradação dos solos e do aumento da emissão de $\mathrm{CO}_{2}$ para atmosfera (SAMBUICHI, 2006).

Apesar de toda essa devastação, o sul da Bahia apresenta ainda uma significativa concentração de árvores nativas em relação a outras regiões da Floresta Atlântica devido ao sistema tradicional de plantio do cacau sob a sombra da floresta raleada, conhecido na região como "cabruca" (SAMBUICHI, 2006). O cacau-cabruca pode ser conceituado como um sistema agrossilvicultural, que se fundamenta na substituição dos elementos do sub-bosque por uma cultura de interesse econômico, implantada sob a proteção das árvores remanescentes de forma descontínua e circundada por vegetação natural, de grande acerto ambiental, estabelecendo relações estáveis com os recursos naturais associados (LOBÃO et al.,1997).

Os primeiros cacauicultores migrantes e imigrantes vindos de regiões áridas do Nordeste e desérticas da Arábia chegaram à região Sul da Bahia há mais de 200 anos e enfrentaram grandes desafios para se estabelecerem agronomicamente, implantando a cacauicultura no sub-bosque da floresta primária (LOBÃO et al., 1997). Inicialmente o cacaueiro foi introduzido sob o dossel florestal, e ao longo dos anos foi sendo adensado, vindo a ocupar todo o sub-bosque. Como espécie humbrófila, o dossel proporcionou-lhe a proteção inicial necessária (LOBÃO et al., 2004). A paisagem antes composta de grandes áreas de floresta nativa cedeu espaço às plantações de cacau que por sua vez serviram de conectores entre os fragmentos de mata remanescentes (OLIVEIRA et al., 2011).

Existem muitos cultivos de cacau associados a outras espécies que lhe conferem sombreamento durante sua fase de desenvolvimento e produtividade. Esse tipo de cultivo, denominado sistema agroflorestal (SAF), promove mudanças ecológicas benéficas ao sistema e tem sido amplamente difundido, por ser um modelo de produção diversificado (SENATORE; MUNÕZ, 2013). A adoção da bananeira nos sistemas agroflorestais cacau cabruca deve-se a diversos aspectos da cultura como adaptação às condições ecológicas destes sistemas, uso como alimento para humanos e animais, elevado valor nutricional, cultura de fácil comércio, excelente fornecedora de sombra para espécies que exigem sombreamento temporário a exemplo do cacau.

A banana é uma das frutas mais importantes a nível mundial, sendo explorada em extensa área na maioria dos países tropicais e subtropicais (DANTAS et al., 2016). O Brasil é o quarto maior produtor 
mundial de banana, com produção de aproximadamente 6,7 milhões de toneladas em 2016 (FAOSTAT, 2018).

Apesar de o Brasil ser um dos principais produtores mundiais de banana, vários fatores limitam seu cultivo, como manejo inadequado, falta de tecnificação e problemas fitossanitários, tais como os nematoides, fungos, dentre outros (ALMEIDA, 2016). Os nematoides Meloidogyne sp., R. similis, $H$. multicinctus, Pratylenchus coffeae e R. reniformis são os mais frequentes na bananicultura brasileira e mundial (WAWERU et al., 2014).

Os fitonematoides constituem uma das principais limitações à cultura, sendo responsáveis por 20\% de queda na produção, chegando a 50\% em algumas situações (RITZINGER et al., 2011).

Infecções causadas por fitonematoides podem interferir no crescimento das plantas, reduzindo o número de raízes funcionais, provocando definhamento e aumento do ciclo, ocorrendo frequentes tombamentos (JESUS; WILCKEN, 2010). Em geral os danos de fitonematoides aos bananais são diretamente proporcionais às suas populações, ocorrendo redução do tamanho, massa e atraso na maturação dos cachos, menor perfilhamento e morte das plantas (NEVES et al., 2009). Neste sentido, o objetivo deste trabalho foi realizar o levantamento de fitonematoides associados à cultura da banana em sistemas agroflorestais cacau cabruca na Região do Sul da Bahia.

\section{Material e métodos}

\section{Coleta das amostras de solo e raízes nas plantas de bananeira}

As coletas foram realizadas em áreas de sistemas agroflorestais cacau cabruca localizados nos municípios de Uruçuca e Itajuípe da região do Sul da Bahia. Foram coletadas, de forma aleatória em caminho zig zag, 1 amostra composta formada por 10 amostras simples em cada área na camada $0-15 \mathrm{~cm}$ de profundidade. As amostras de solo e raízes após coletadas, foram secas ao ar, destorroadas, homogeneizadas, peneiradas em peneira de $2 \mathrm{~mm}$, e em seguida armazenadas a $4^{\circ} \mathrm{C}$ em câmara fria, até realização das análises (Figura 1). 
Figura 1 - Coleta de amostras de solo e raízes em áreas de sistemas agroflorestais cacau cabruca localizados na região do Sul da Bahia. Uruçuca, 2019.
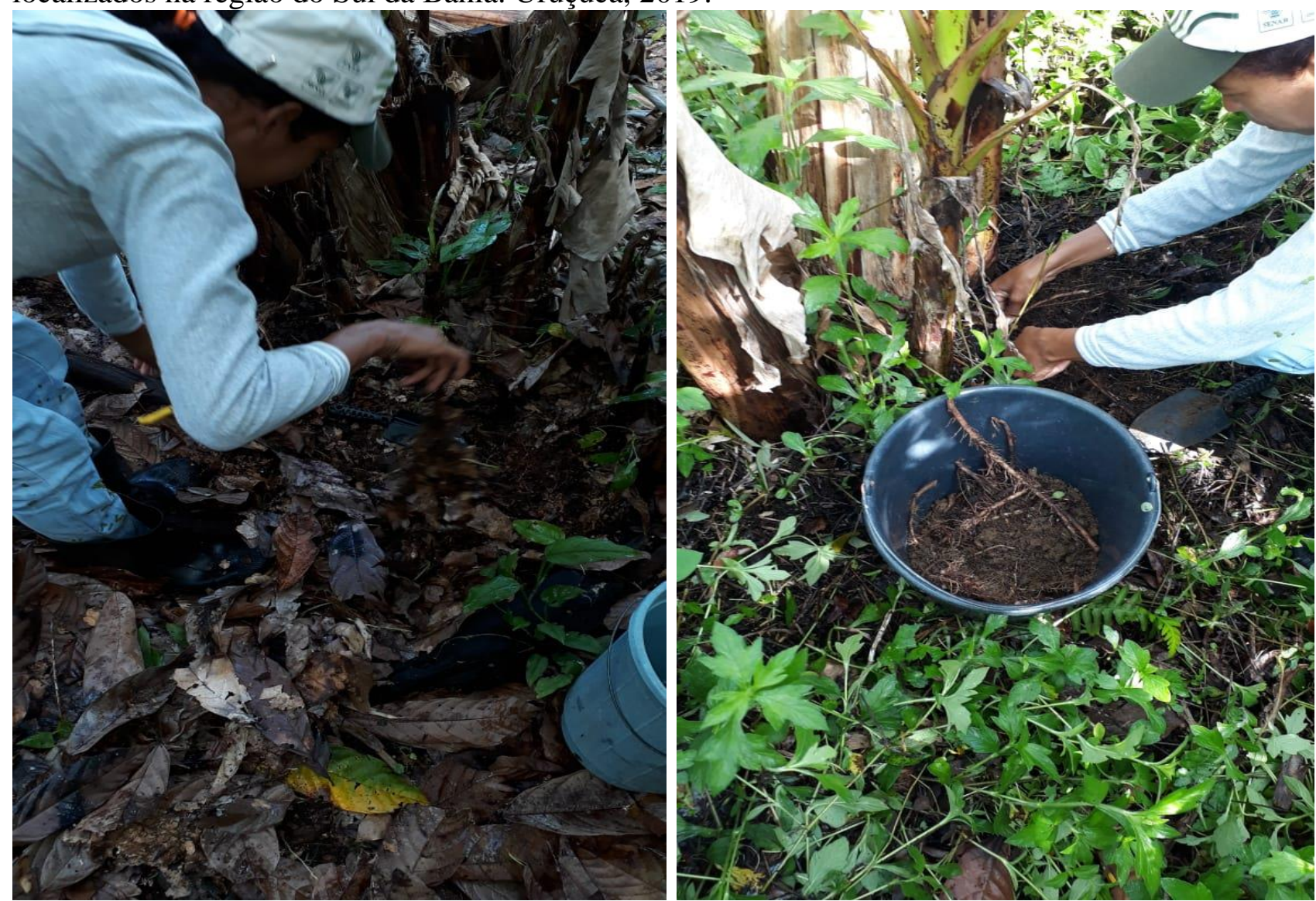

Fonte: Ronildo Santos Silva, 2019

\section{Extração, quantificação e identificação das espécies de nematoides}

\section{Extração de nematoides no solo}

As amostras de solo $\left(250 \mathrm{~cm}^{3}\right)$, foram processadas pelo método de peneiramento sucessivo em água com peneiras de malhas abertas em $0,85 \mathrm{~mm}, 0,35 \mathrm{~mm}$ e $0,025 \mathrm{~mm}$ respectivamente, seguido de centrifugação em água a $3000 \mathrm{rpm}$ por 5 min, e depois em solução de sacarose (50\%) a $1500 \mathrm{rpm}$ por 1 min (JENKINS, 1964).

\section{Extração de nematoides nas raízes}

A extração dos nematoides das raízes foi realizada conforme metodologia proposta por Coolen \& D’Herde (1972), onde em um copo de liquidificador, foi adicionada $10 \mathrm{~g}$ de raízes, juntamente com $500 \mathrm{ml}$ de água e caulim, e em seguida, trituradas por 30 segundos. A suspensão obtida foi peneirada em malhas com abertura de $0,85 \mathrm{~mm}, 0,35 \mathrm{~mm}$ e $0,025 \mathrm{~mm}$ respectivamente para obtenção dos nematoides. Os nematoides extraídos foram concentrados em $5 \mathrm{ml}$ de água $+5 \mathrm{ml}$ de solução de formalina a 10\%, para preservação. A identificação e contagem dos nematoides foram realizadas em microscópio com auxílio de chave taxonômica e câmara de Peters. 


\section{Análise estatística}

Foi calculada a abundância relativa das espécies de nematoides nas amostras de solo e nas raízes, utilizando a fórmula $\mathrm{Ar} \%=\mathrm{A} \times 100 / \mathrm{N}$, onde: $\mathrm{A}=$ número de indivíduos de uma mesma espécie na amostra e $\mathrm{N}=$ número total de nematoides na amostra. Foi calculada também a frequência relativa das espécies de nematoides, através da fórmula: $\mathrm{Fr} \%=$ na x 100 / NA, onde: na $=$ o número de amostras em que a espécie de nematoide ocorreu; $\mathrm{NA}=$ o número total de amostras coletadas na área.

\section{Resultados e discussão}

Nas amostras de solo e raízes de bananeira coletadas nas áreas de cacau cabruca, foram identificadas as espécies de fitonematoides $H$. multicinctus, R. reniformis, R. similis e nematoides de vida livre (Tabela 1).

Tabela 1. Número médio de espécies de fitonematoides associados à cultura da banana em sistemas agroflorestais cacau cabruca nos municípios de Itajuípe-Bahia e Uruçuca-Bahia, 2019.

\begin{tabular}{|c|c|c|c|c|c|c|c|c|c|c|}
\hline \multirow{3}{*}{$\begin{array}{l}\text { Locais de } \\
\text { coleta }\end{array}$} & \multicolumn{10}{|c|}{ Espécies de nematoides } \\
\hline & \multicolumn{2}{|c|}{$\begin{array}{c}\text { Helicotylenchulus } \\
\text { multicinctus }\end{array}$} & \multicolumn{2}{|c|}{$\begin{array}{l}\text { Rotylenchulus } \\
\text { reniformis }\end{array}$} & \multicolumn{2}{|c|}{$\begin{array}{l}\text { Radopholus } \\
\text { similis }\end{array}$} & \multicolumn{2}{|c|}{$\begin{array}{l}\text { Nematoide de } \\
\text { vida livre }\end{array}$} & \multicolumn{2}{|c|}{ Total } \\
\hline & raiz* & solo** & raiz & solo & raiz & solo & raiz & solo & raiz & solo \\
\hline Uruçuca 1 & 240 & 300 & - & - & - & - & 920 & 760 & 1160 & 1060 \\
\hline Uruçuca 2 & 450 & 280 & - & - & - & - & 800 & 500 & 1250 & 780 \\
\hline Uruçuca 3 & 640 & 230 & - & - & - & - & 1480 & 200 & 2120 & 430 \\
\hline Uruçuca 4 & 300 & 280 & - & - & - & - & 840 & 1240 & 1140 & 1520 \\
\hline Uruçuca 5 & 1080 & 400 & - & - & - & - & 640 & 450 & 1720 & 850 \\
\hline Uruçuca 6 & 800 & 520 & - & - & - & - & 920 & 840 & 1720 & 1360 \\
\hline Uruçuca 7 & 400 & 360 & - & - & - & 10 & 680 & 230 & 1090 & 590 \\
\hline Uruçuca 8 & 200 & 150 & - & 10 & - & - & 500 & 380 & 700 & 530 \\
\hline Itajuípe 1 & 170 & 120 & - & 30 & - & - & 520 & 540 & 690 & 690 \\
\hline Itajuípe 2 & 40 & 30 & 20 & 40 & - & - & 300 & 740 & 360 & 810 \\
\hline
\end{tabular}

*População média por $10 \mathrm{~g}$ de raízes; ** População média por $250 \mathrm{~cm}^{3}$ de solo.

O fitonematoide $H$. multicinctus foi registrado em todas as áreas amostradas, sendo a maior população total observada nas amostras de solo coletadas na área Uruçuca $6\left(520\right.$ nematoides $/ 250 \mathrm{~cm}^{3}$ de solo), enquanto que o maior número deste nematoide em raízes foi registrado em Uruçuca 5 (1080 nematoides $/ 10 \mathrm{~g}$ de raízes). Apesar de ser considerado como patógeno secundário, esse nematoide ainda não tem sua importância bem definida como causador de danos à cultura da banana no Brasil (DIAS et al., 2011). É comumente encontrado em associação com R. similis e/ou Meloidogyne spp., porém pouco se conhece sobre a sua importância na cultura da banana no Brasil (KUBO et al., 2005). Os sintomas causados por $H$. multicinctus consistem em pequenas lesões acastanhadas com aparência de pontuações 
superficiais, principalmente nas raízes mais grossas. Em condições de intenso ataque, podem coalescer, dando um aspecto necrosado às raízes (DIAS-ARIEIRA; COSTA, 2008).

O nematoide R. reniformis foi registrado em amostras de solo coletadas nas áreas Uruçuca 8, Itajuípe 1 e Itajuípe 2, com populações de respectivamente 10, 30 e 40 nematoides $/ 250 \mathrm{~cm}^{3}$ de solo. Esta espécie de nematoide foi registrada apenas em amostras de raízes de bananeira coletadas na área Itajuípe 2 (20 nematoides/10g de raízes). O gênero Rotylenchulus compreende 11 espécies válidas, sendo caracterizados como semi-endo parasitos de inúmeras espécies de plantas e encontram-se distribuídos principalmente em regiões tropicais e subtropicais (PALOMARES-RIUS et al., 2017).

Apenas em amostras de solo coletadas em sistema cacau cabruca na área Uruçuca 7, foram identificados o nematoide $R$. similis (10 nematoides $/ 250 \mathrm{~cm}^{3}$ de solo). Dentre as diversas espécies de fitonematoides que ocorrem na bananicultura, R. similis, conhecido como nematoide cavernícola, assume papel de destaque por causar maior dano à cultura (QUÉNÉHERVÉ, 2009). Seu principal dano econômico deriva das necroses radiculares, que resultam no enfraquecimento do seu poder de sustentação mecânica (OLIVEIRA et al., 2015). A presença deste patógeno na cultura da bananeira impede o funcionamento satisfatório do sistema radicular das plantas, limitando a absorção de água e de nutrientes. Isso ocorre em consequência das lesões geradas no córtex das raízes pela presença dos indivíduos que se instalam e que alimentam das células do córtex (ELSEN et al., 2002).

Foram registrados nematoides de vida em todas as amostras de solo e raízes coletadas nas áreas de cacau cabruca (Tabela 1). Os nematoides de vida livre diferentemente dos nematoides fitoparasitas são considerados benéficos à agricultura e podem influenciar na nutrição, qualidade do solo, vivendo associados a raízes sem causar danos às culturas, além de atuarem no controle biológico e serem bons indicadores na avaliação da qualidade do solo. Os índices das comunidades de nematoides de vida livre no solo (taxa de ocorrência, abundância e diversidade) são essenciais para detectar impactos sobre diferentes tipos de manejo dos solos, bem como distúrbios que eles sofrem (RITZINGER et al., 2010).

O nematoide $H$. multicinctus apresentou frequência relativa de 100\% nas amostras de solo e raízes de banana nos sistemas cacau cabruca. Entretanto, foi verificada baixa frequência dos nematoides $R$. reniformis (30\% no solo e 10\% nas raízes) e R. similis (10\% nas raízes) (Tabela 2). Em um levantamento dos fitonematoides associados aos cultivos de bananeiras em 12 municípios da região sul da Bahia, Oliveira et al., (2015), também verificaram alta frequência relativa de $H$. multicinctus, variando 87 a $90 \%$.

Com relação à abundância relativa das espécies de nematoides (Tabela 3), verifica-se que embora o nematoide $H$. multicinctus tenha sido registrado em todas as áreas, os maiores valores foram registrados nas amostras de solo coletadas na área Uruçuca 5 (46,5\%) e nas amostras de raízes da área Uruçuca 7 (61,0\%). Além da planta hospedeira, fatores como características químicas e físicas do solo, práticas de 
manejo, condições edafoclimáticas, sistemas de cultivo, dentre outros influenciam na densidade populacional e diversidade de espécies de fitonematoides presentes no solo.

Tabela 2. Frequência relativa (Fr) das espécies de fitonematoides associados à cultura da banana em sistemas agroflorestais cacau cabruca nos municípios de Itajuípe-Bahia e Uruçuca-Bahia, 2019.

\begin{tabular}{lcc}
\hline \multicolumn{1}{c}{ Nematoides ocorrentes } & Solo & Raízes \\
\hline Helicotylenchus multicinctus & 100 & 100 \\
Rotylenchulus reniformis & 30 & 10 \\
Radopholus similis & 10 & - \\
Nematoide de vida livre & 100 & 100 \\
\hline
\end{tabular}

Tabela 3. Abundância relativa (Ar) de nematoides de diferentes associados à cultura da banana em sistemas agroflorestais cacau cabruca nos municípios de Itajuípe-Bahia e Uruçuca-Bahia, 2019.

\begin{tabular}{clcc}
\hline Locais & \multicolumn{1}{c}{ Nematoides ocorrentes } & Solo & Ar (\%) \\
& & 20,7 & Raízes \\
\hline Uruçuca 1 & Helicotylenchulus multicinctus & 36,0 & 28,3 \\
Uruçuca 2 & Helicotylenchulus multicinctus & 30,2 & 35,9 \\
Uruçuca 3 & Helicotylenchulus multicinctus & 26,3 & 53,5 \\
Uruçuca 4 & Helicotylenchulus multicinctus & 62,8 & 18,4 \\
Uruçuca 5 & Helicotylenchulus multicinctus & 46,5 & 47,1 \\
Uruçuca 6 & Helicotylenchulus multicinctus & 36,7 & 38,2 \\
Uruçuca 7 & Helicotylenchulus multicinctus & - & 61,0 \\
& Radopholus similis & 28,6 & 1,7 \\
Uruçuca 8 & Helicotylenchulus multicinctus & - & 28,3 \\
& Rotylenchulus reniformis & 17,4 & 1,9 \\
Itajuípe 1 & Helicotylenchulus multicinctus & - & 17,4 \\
& Rotylenchulus reniformis & 3,7 & 4,3 \\
Itajuípe 2 & Helicotylenchulus multicinctus & 5,6 & 3,7 \\
& Rotylenchulus reniformis & 4,9 \\
\hline
\end{tabular}

\section{Conclusão}

Os fitonematoides associados à cultura da bananeira em sistemas agroflorestais cacau cabruca localizados na região Sul da Bahia nos municípios de Itajuípe são H. multicinctus e R. reniformis e em Uruçuca: H. multicinctus, R. reniformis, R. similis.

As maiores populações presentes nas amostras de solo e de raízes coletadas nas áreas são do fitonematoide $H$. multicinctus.

Estudos futuros devem ser realizados para avaliar o efeito de características químicas e físicas destes solos e de fatores edafoclimáticos sobre as populações de fitonematoides, bem como, definir práticas de manejo a serem adotadas pelos agricultores da região. 


\section{Agradecimentos}

Os autores agradecem ao Instituto Federal de Educação, Ciência e Tecnologia Baiano - IFBaiano pelo financiamento do projeto aprovado na Chamada Interna PROPES N02/2018 e à concessão de bolsa de produtividade à profa. Dra. Carla da Silva Sousa e ao Conselho Nacional de Desenvolvimento Científico e Tecnológico - CNPq pela concessão da bolsa de iniciação científica à discente Joseane Santos.

\section{Referências bibliográficas}

ALMEIDA, N. O. Ocorrência de nematoides na cultura da banana no estado de Goiás e sua correlação com o mal-do-Panamá e com fatores edáficos. 2016. 57 f. Dissertação (Mestrado em Agronomia) - Universidade Federal de Goiás, Goiânia, 2016.

COOLEN, W. A.; D'HERDE, C. J. A method for the quantitative extraction of nematodes from plant tissue. Ghent, State Agricultural Research Center. 77p. 1972.

DANTAS, J. L. L.; SILVA, S. de O. e; SOARES FILHO, W. dos S.; CARVALHO, P. C. L de. Filogenia, história, evolução, distribuição geográfica e habitat. In: FERREIRA, C. F.; SILVA, S. de O. e; AMORIM, E. P.; SANTOS-SEREJO, J. A. dos. (Ed.). O Agronegócio da Banana. Brasília, DF: Embrapa, 2016. p. 15-28.

DIAS, J. R. et al. 2011. Levantamento de nematoides em bananais do norte de Minas. In Fórum de Ensino, Pesquisa, Extensão e Gestão, 5. Montes Claros, Unimontes. Disponível em: <http:// www.fepeg.unimontes.br/index>. Acesso em: abr. 2020.

DIAS-ARIEIRA, C.R.; COSTA, A.T. Nematoides causadores de doenças em frutíferas. Agro@mbiente On-line, v.2, n.1, p.46-56, 2008.

ELSEN, A.; STOFFELEN, R.; THI TUYET, N.; BAIMEY, H.; BOULOIS, H. D.; WAELE, D. D. In vitro screening for resistance to Radopholus similis in Musa spp. Plant Science. v. 163, p. 407- 416, 2002.

FAOSTAT - Food And Agriculture Organization of the United Nations Statistics Disponível em: <http://www.fao.org/faostat/en/\#data/QC>. Acesso em: 20 ago. 2018.

JENKINS, W. R. A rapid centrifugal-flotation technique for separating nematodes from soil. Plant Disease Reporter, v. 48, n. 9, p. 692, 1964.

JESUS, A.M.; WILCKEN, R.S. Reprodução de Meloidogyne incognita, M. javanica e Pratylenchus coffeae em diferentes cultivares de bananeira. Nematologia Brasileira, v.34, n.1, p. 3-9, 2010.

KUBO, R. K. et al. 2005. Nematoides fitoparasitos da bananeira. In: Reunião Itinerante de Fitossanidade do Instituto Biológico, São Paulo, Instituto Biológico. Disponível em: <http:// www.biologico.sp.gov.br/rifib/XIII\%20RIFIB/ kubo.pdf>. Acesso em: abr. 2020.

LOBÃO, D. E.; SETENTA W. C.; VALLE, R. R. Sistema agrossilvicultural cacaueiro - modelo de agricultura sustentável. Agrossilvicultura, v. 1, n. 2, p. 163-173, 2004. 
LOBÃO, D. E.; SETENTA, W. C.; LOBÃO, E. S. P.; CURVELO, K.; VALLE, R. R. Cacau Cabruca sistema agrossilvicultural tropical. In: VALLE, R. R. (Ed.). Ciência, tecnologia e manejo do cacaueiro. Itabuna: Gráfica e Editora Vital, p. 290-323, 2007.

NEVES, W.S.; DIAS, M.C.S.; BARBOSA, J.G. Flutuação populacional de nematoides em bananais de Minas Gerais e da Bahia (anos 2003 a 2008). Nematologia Brasileira, v.33, n.4, p. 281-285, 2009.

OLIVEIRA, U.L.C.; SILVEIRA, A.J.; SOARES, P.L.M.; BITTENCOURT, A.L. Fitonematoides associados a cultivos de bananeiras na região Sul da Região. Agrotrópica, v.27, n. 1, p. 5-14. 2015.

OLIVEIRA, R.M.; COSTA, W.R.C.; SAMBUICHI, R.S.; HELLMEISTER JUNIOR, P. Importância do sistema agroflorestal cacau cabruca para a conservação florestal da região cacaueira, Sul da Bahia, Brasil. Revista Geográfica de América Central, Número especial, p. 1-12, 2011.

PALOMARES-RIUS. J. E.; CANTALAPIEDRA-NAVARRETE, C.; ARCHIDONA-YUSTE, A.; TZORTZAKAKIS, E.A.; BIRMPILIS, I.G.; VOVLAS, N.; SUBBOTIN, S.A.; CASTILLO, P. Prevalenceand molecular diversity of reniform nematodes of the genus Rotylenchulus (Nematoda: Rotylenchulinae) in the Mediterranean Basin. European Journal of Plant Pathology, v.150, n. 2, p.439455, 2017.

QUÉNÉHERVÉ, P. Integrated management of banana nematodes. In: Ciancio A, Mukerji KG (eds.), Integrated Management of Fruit Crops and Forest Nematodes. Springer, The Netherlands, p. 3-61. 2009.

RITZINGER, C. H.; FANCELLI, S. P.; RITZINGER, M. R. Nematoides: Bioindicadores de sustentabilidade e mudanças edafoclimáticas. Revista Brasileira de Fruticultura, v. 32, n. 4, p. 12891296, 2010.

RITZINGER, C.H.P.; FANCELLI, M.; CORDEIRO, Z.J.M.; VIEIRA, R.S.; LEDO, C.A.S. Avaliação da população de nematoides em bananal com e sem o uso de organomineral. Revista Brasileira de Fruticultura, v.33, n.4, p. 1103-1110, 2011.

SAMBUICHI, R.H.R. Estrutura e dinâmica do componente arbóreo em área de cabruca na região cacaueira do sul da Bahia, Brasil. Acta Botânica Brasílica, v.20, n.4, p.943-954, 2006.

SENATORE, G.; MUÑOZ, A. I. Produção de cacau, panorama da cultura. Mercado \& Negócios, Agroanalysis. p. 26, 2013.

SILVA, M. B.L.; RAMOS, A.M. Composição química, textura e aceitação sensorial de doces em massa elaborados com polpa de banana e banana integral. Revista Ceres, v. 56, n.5, p. 551-554, 2009.

WAWERU, B.; TUROOP, L.; KAHANGI, E.; COYNE, D.; DUBOIS, T. Non-pathogenic Fusarium oxysporum endophytes provide field control of nematodes, improving yield of banana (Musa sp.). Biological Control.v.74, p. 82-88. 2014. 


\begin{tabular}{|c|c|}
\hline Informações do Artigo & Article Information \\
\hline Recebido em: 10/05/2020 & Received on: $21 / 05 / 2020$ \\
\hline Aceito em: 20/06/2020 & Accepted in: 20/06/2020 \\
\hline Publicado em: 26/06/2020 & Published on: $26 / 06 / 2020$ \\
\hline $\begin{array}{l}\text { Conflitos de Interesse: Os autores declaram não } \\
\text { haver quaisquer conflitos de interesse referente a } \\
\text { este artigo. }\end{array}$ & Conflict of Interest: No reported. \\
\hline $\begin{array}{l}\text { Como citar este artigo } \\
\text { Sousa, C., Silva, J., \& Lima, Érica. (2020). } \\
\text { Fitonematoides associados à cultura da banana em } \\
\text { sistemas cacau cabruca. Revista Macambira, 4(1), } \\
\text { e041002. https://doi.org/10.35642/rm.v4i1.464 }\end{array}$ & $\begin{array}{l}\text { How to cite this article } \\
\text { Sousa, C., Silva, J., \& Lima, Érica. (2020). } \\
\text { Phytonematoids associated with banana culture in } \\
\text { cocoa cabruca systems. Revista Macambira, 4(1), } \\
\text { e041002. https://doi.org/10.35642/rm.v4i1.464 }\end{array}$ \\
\hline $\begin{array}{l}\text { Licença: } \\
\qquad \text { cc) (†)(9) }\end{array}$ & (c) (†) \\
\hline $\begin{array}{l}\text { Este trabalho está licenciado sob uma Licença } \\
\text { Internacional Creative Commons Attribution- } \\
\text { NonCommercial-ShareAlike } 4.0 \text { International. }\end{array}$ & $\begin{array}{l}\text { This work is licensed under a Creative Commons } \\
\text { Attribution-NonCommercial-ShareAlike } \\
\text { International License. }\end{array}$ \\
\hline
\end{tabular}

